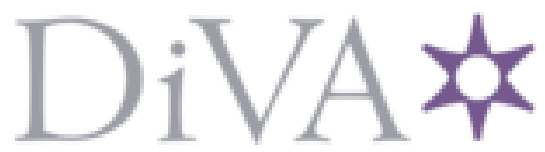

http://www.diva-portal.org

This is the published version of a paper published in JOURNAL OF PHYSICAL CHEMISTRY A.

Citation for the original published paper (version of record):

Brinck, T., Carlqvist, P., Halldin Stenlid, J. (2016)

Local Electron Attachment Energy and Its Use for Predicting Nucleophilic Reactions and

Halogen Bonding.

JOURNAL OF PHYSICAL CHEMISTRY A, 120(50): 10023-10032

https://doi.org/10.1021/acs.jpca.6b10142

Access to the published version may require subscription.

N.B. When citing this work, cite the original published paper.

Permanent link to this version:

http://urn.kb.se/resolve?urn=urn:nbn:se:kth:diva-200209 


\title{
Local Electron Attachment Energy and Its Use for Predicting Nucleophilic Reactions and Halogen Bonding
}

\author{
Tore Brinck, ${ }^{*}$ (1) Peter Carlqvist, ${ }^{\dagger}$ and Joakim H. Stenlid \\ Applied Physical Chemistry, School of Chemical Science and Engineering, KTH Royal Institute of Technology, SE-100 44 \\ Stockholm, Sweden
}

\section{Supporting Information}

ABSTRACT: A new local property, the local electron attachment energy $[E(\mathbf{r})]$, is introduced and is demonstrated to be a useful guide to predict intermolecular interactions and chemical reactivity. The $E(\mathbf{r})$ is analogous to the average local ionization energy but indicates susceptibility toward interactions with nucleophiles rather than electrophiles. The functional form $E(\mathbf{r})$ is motivated based on Janak's theorem and the piecewise linear energy dependence of electron addition to atomic and molecular systems. Within the generalized Kohn-Sham method (GKS-DFT), only the virtual orbitals with negative eigenvalues contribute to $E(\mathbf{r})$. In the present study, $E(\mathbf{r})$ has been computed from orbitals obtained from GKS-DFT computations with a hybrid exchangecorrelation functional. It is shown that $E(\mathbf{r})$ computed on a molecular isodensity surface, $E_{S}(\mathbf{r})$, reflects the regioselectivity and relative reactivity for nucleophilic aromatic substitution, nucleophilic addition to activated double bonds, and formation of halogen bonds. Good to excellent correlations between experimental or theoretical measures of interaction strengths and minima in $E_{S}(\mathbf{r})\left(E_{S, \min }\right)$ are demonstrated.

\section{INTRODUCTION}

Local molecular properties computed on molecular surfaces have been used extensively to analyze and predict intermolecular interactions and chemical reactivity. ${ }^{1-8}$ The molecular electrostatic potential $[V(\mathbf{r})]$ has been the dominating property in such analysis, and the predictive power of the surface $V(\mathbf{r})$ [labeled $\left.V_{\mathrm{S}}(\mathbf{r})\right]$ for noncovalent interactions, such as hydrogen bonding and halogen bonding, is well documented. ${ }^{6,9-15}$ One limitation of $V_{\mathrm{S}}(\mathbf{r})$ is that it only reflects the susceptibility toward electrostatic interaction, and thus it is less well suited to analyze the propensity for interactions that involve a significant degree of redistribution of the charge densities of the interacting species, such as donor-acceptor interactions and the formation of covalent bonds. To increase the applicability of the local surface property approach, Sjoberg et al. devised the average local ionization energy $[I(\mathbf{r})]$ as a complement to $V(\mathbf{r})$ for analyzing susceptibility toward electrophilic attack. ${ }^{2}$ The $\bar{I}(\mathbf{r})$ is rigorously defined within Hartree-Fock (HF) theory and Kohn-Sham density functional theory (KS-DFT) by

$$
\bar{I}(\mathbf{r})=\frac{\sum_{i}^{\mathrm{occ}}-\varepsilon_{i} \rho_{i}(\mathbf{r})}{\rho(\mathbf{r})}
$$

where $\varepsilon_{i}$ is the energy of orbital $i, \rho_{i}(\mathbf{r})$ is the density of the orbital, and $\rho(\mathbf{r})$ is the total electron density. According to Koopman's theorem, ${ }^{16}$ the absolute values of the orbital energies are approximations to the ionization energies, and $\bar{I}(\mathbf{r})$ can be interpreted as the average energy needed to ionize an electron at

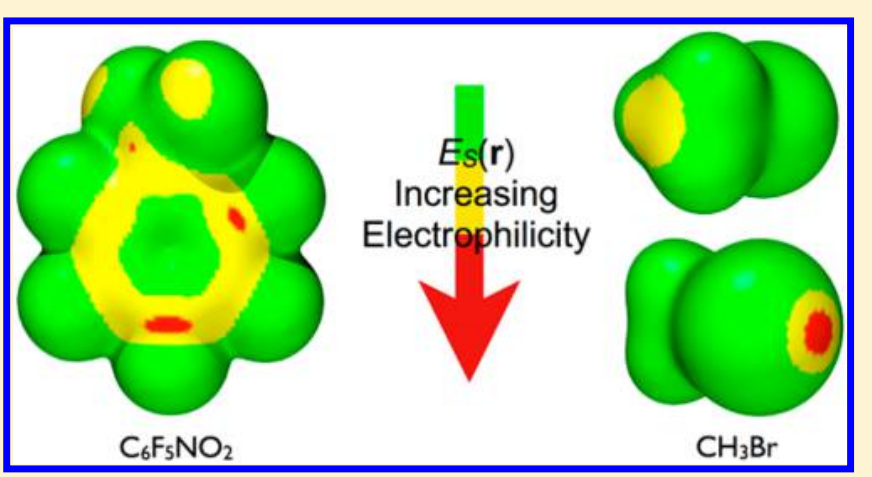

a point $\mathbf{r}$ in the space of a molecule or atom. Bulat et al. have shown that $\bar{I}(\mathbf{r})$ is invariant to an unitary orbital transformation and that $\bar{I}(\mathbf{r})$ can be written as a function of the local electron temperature $[T(\mathbf{r})], V(\mathbf{r})$, and the exchange-correlation potential $\left[V_{\mathrm{XC}}(\mathbf{r})\right]$. (In the case of a HF determinant, $V_{\mathrm{XC}}(\mathbf{r})$ equals the Slater potential. $)^{17}$

$$
\bar{I}(\mathbf{r})=-\frac{3}{2} k T(\mathbf{r})+V(\mathbf{r})-V_{\mathrm{XC}}(\mathbf{r})
$$

The contribution of $k T(\mathbf{r})$ is particularly interesting because it shows that $\bar{I}(\mathbf{r})$ depends not only on the local potential felt by an electron at $\mathbf{r}$ but also on the local kinetic energy density, that is, $3 k T(\mathbf{r}) / 2=t_{\mathrm{S}}(\mathbf{r}) / \rho(\mathbf{r})$, where $t_{\mathrm{S}}(\mathbf{r})$ is the local kinetic energy density and is obtained as $t_{\mathrm{S}}(\mathbf{r})=-1 / 2 \sum_{i}^{\text {occ }} \psi_{i}^{*}(\mathbf{r}) \nabla^{2} \psi_{i}(\mathbf{r}) .^{17}$

For the purpose of analyzing chemical reactivity, $\bar{I}(\mathbf{r})$ is normally computed on molecular surfaces defined by a constant electron density and is then labeled $\bar{I}_{\mathrm{S}}(\mathbf{r})$. On such a surface, the denominator in eq 1 is constant, and $\bar{I}_{S}(\mathbf{r})$ reflects the varying ease of ionization over the surface. The positions where $\bar{I}_{S}(\mathbf{r})$ has its lowest values, the local surface minima $\left(\bar{I}_{S, \text { min }}\right)$, are indicative of sites that are prone to interact with electrophiles. In contrast with $V_{S}(\mathbf{r}), \bar{I}_{S}(\mathbf{r})$ has been found to reflect a molecule's tendency not only for electrostatic interaction but also for charge transfer

Received: October 7, 2016

Revised: November 24, 2016

Published: November 29, 2016 
and polarization. ${ }^{5,8,11} \bar{I}_{\mathrm{S}}(\mathbf{r})$ has been shown to be particularly well-suited to describe reactivity toward electrophiles in solution, where electrostatic interactions are partly shielded and their importance is reduced. For example, $\bar{I}_{S}(\mathbf{r})$ is an effective tool for the prediction of regioselectivity and relative reactivity of aromatic substrates toward electrophilic aromatic substitution. ${ }^{2,18-20}$ Another use of $\bar{I}_{\mathrm{S}}(\mathbf{r})$ has been in the analysis of aqueous basicity; the most basic sites in neutral molecules and anions generally have associated $\bar{I}_{\mathrm{S}, \text { min }}$, and the $\bar{I}_{\mathrm{S} \text {,min }}$ values often correlate well with the $\mathrm{p} K_{\mathrm{a}}$ values of the conjugate acids within series of congeneric molecules. ${ }^{5,18,21}$

In contrast with the $V_{S}(\mathbf{r})$, which attains both negative and positive values, $\bar{I}_{\mathrm{S}}(\mathbf{r})$ cannot be used to characterize susceptibility for nucleophilic attack. In an attempt to develop a corresponding surface property to $\bar{I}_{S}(\mathbf{r})$ for interactions with nucleophiles, Clark and coworkers defined the local electron affinity $\left[E A_{\mathrm{L}}(\mathbf{r})\right]$ using an analogous expression in terms of the virtual orbitals. 7,22

$$
E A_{\mathrm{L}}(\mathbf{r})=\frac{\sum_{i=\mathrm{LUMO}}^{\text {norbs }}-\varepsilon_{i} \rho_{i}(\mathbf{r})}{\sum_{i=\mathrm{LUMO}}^{\text {norbs }} \rho_{i}(\mathbf{r})}
$$

The use of the virtual orbital energies for estimating electron affinities can be justified by Koopman's theorem, although there is no error cancellation between orbital relaxation and electron correlation effects, as is typical for ionization energies. However, there are other issues with $E A_{\mathrm{L}}(\mathbf{r})$ that need to be considered. First of all, it can be noted that $E A_{\mathrm{L}}(\mathbf{r})$ is very sensitive to the size of the basis set, as the number of virtual orbitals and their energies depend on the number and type of basis functions. Clark has mostly used $E A_{\mathrm{L}}(\mathbf{r})$ together with semiempirical methods, and because these generally employ a minimum basis set description the basis set issue is less severe. To use semiemprical methods with an explicit $\mathrm{d}$ orbital description, he has devised a method to identify which orbitals to include when computing $E A_{\mathrm{L}}(\mathbf{r})$ with a nonminimal basis set. ${ }^{22}$ However, even when using a virtual orbital space corresponding to only the valence orbitals, highenergy orbitals, which have little influence on the reactivity, can contribute significantly to the $E A_{\mathrm{L}}(\mathbf{r})$. For example, an $E A_{\mathrm{L}}(\mathbf{r})$ analysis of an aromatic system typically finds the aromatic carbons to be relatively unreactive due to the contributions of high-energy virtual $\sigma^{*}$ orbitals.

Another problem with $E A_{\mathrm{L}}(\mathbf{r})$ as a local surface property is that the denominator in eq 3 is defined by the virtual orbital density and thus varies over an isodensity surface. The susceptibility for electrophilic attack depends not only on the energies of low-lying virtual orbitals but also on the magnitude of their combined densities. However, with the current expression (eq 3), regions with low total virtual orbital density are often identified as highly reactive.

It should be recognized that $E A_{\mathrm{L}}(\mathbf{r})$ has successfully been used in a number of applications, including as a descriptor in QSAR analysis for drug design and for the calculation of chargetransport properties of organic-field transistors. ${ }^{23-25}$ On the contrary, the use of $E A_{\mathrm{L}}(\mathbf{r})$ in the analysis of chemical reactivity has been relatively sparse, which potentially can be traced to some of the issues discussed above.

The objective of the current work has been to define a new local surface property that is tuned for predicting chemical reactivity and intermolecular interactions and that is wellbehaved with large basis sets and more exact KS-DFT methods. We will introduce the local electron attachment energy, $E(\mathbf{r})$, which can be seen as a local reactivity descriptor. The capacity of the surface $E(\mathbf{r})$, labeled as $E_{S}(\mathbf{r})$, for predicting the local and global reactivities for a number of chemical systems that interact with nucleophiles in various interaction types has been analyzed. As will be shown, $E_{S}(\mathbf{r})$ has a predictive capacity that is comparable to $\bar{I}_{\mathrm{S}}(\mathbf{r})$ for the corresponding processes with electrophiles, and $E_{S}(\mathbf{r})$ is found to provide information that in many cases is complementary to $V_{S}(\mathbf{r})$.

\section{THEORY}

In a first effort to modify $E A_{\mathrm{L}}(\mathbf{r})$ and to obtain a local property that more closely corresponds to the $\bar{I}(\mathbf{r})$, we have defined the expression

$$
E^{E_{0}}(\mathbf{r})=\frac{\sum_{i=\mathrm{LUMO}}^{\varepsilon_{i}<E_{0}}\left(\varepsilon_{i}-E_{0}\right) \rho_{i}(\mathbf{r})}{\rho(\mathbf{r})}
$$

The first difference compared to $E A_{\mathrm{L}}(\mathbf{r})$, is that the denominator is defined by the ground state density rather than the virtual orbital density as in eq 3. This definition is connected to the intended use of $E^{E_{0}}(\mathbf{r})$ as a local surface property. The use of surface properties for analyzing chemical reactivity is based on the assumption that the short-range repulsive potential, which depends on electron-electron interactions, is nearly constant over an isodensity surface; thus the varying reactivity over the surface will be defined by the variations in the attractive potential expressed by the local property. To fulfill this criterion, the denominator needs to be constant at constant $\rho(\mathbf{r})$, such that $E^{E_{0}}(\mathbf{r})$ at each point on the surface has an energy contribution $\left(\varepsilon_{i}\right.$ $-E_{0}$ ) from orbital $i$ that is proportional to the orbital density at that point. Using a denominator that is a summation of the densities of the contributing virtual orbitals, as in eq 3 , will significantly reduce the regioselectivity information that is provided by the local property; this is particularly obvious if only one virtual orbital contributes, since the value of the local property then will be the same everywhere.

The second difference is the introduction of the energy offset, $E_{0}$, such that only orbitals with an energy lower than this value are considered. This is not a cutoff in the normal sense, as the contribution from an orbital to the overall $E^{E_{0}}(\mathbf{r})$ value smoothly approaches zero as $\varepsilon_{i}$ approaches $E_{0}$. The need for an energy criterion for selecting the active orbitals is obvious, as only the lowest-lying virtual orbitals are expected to contribute to the reactivity and accept electron transfer from donor orbitals. At first glance the selection of the $E_{0}$ value is more difficult. After all, virtual orbital energies are strongly method-dependent; for example, virtual orbital energies obtained by HF are generally much more positive than those obtained with KS-DFT using a local density approximation (LDA). Rather than attempting to optimize a method-dependent $E_{0}$, we will first determine the best theoretical value at the most exact DFT level.

The starting point for the derivation of $E_{0}$ will be Janak's theorem, which states that the derivative of the total energy with respect to the orbital occupancy is equal to the corresponding orbital energy. ${ }^{26}$

$$
\frac{\partial E}{\partial n_{i}}=\varepsilon_{i}
$$

This theorem holds for a local exchange correlation potential as well as for a nonlocal potential in the case of the generalized Kohn-Sham (GKS-DFT) method; that is, it is applicable to local LDA as well as hybrid methods, invoking HF exchange and even regular HF. ${ }^{27,28}$ The theorem shows that only virtual orbitals with a negative orbital energy will bind a fractional electron, $\Delta n_{i}{ }^{28}$ and that the binding energy is $\Delta n_{i} \varepsilon_{i}$ when $\Delta n_{i}$ is infinitely 
small. Furthermore, it is known that the exact energy as a function of fractional electron occupancy is always linear between integer electron occupancies for any atomic or molecular system. ${ }^{27,29}$ This criterion is normally not fulfilled for approximate DFT methods, and LDA and GGA methods typically give convex curves, whereas the HF curve is concave. $^{27,29}$ However, for an exact GKS-DFT method that fulfills the linearity criterion, the energy change upon the addition of a fractional electron is given by

$$
E(M+\Delta N)-E(M)=\Delta N \varepsilon_{\text {LUMO }}
$$

and the electron affinity $E A=-\varepsilon_{\text {LUMO }} \cdot{ }^{27,28}$ We can expect that such a method that provides accurate electron affinities and energies of charge-transfer interactions will provide reliable energies for fractional addition to other orbitals as well by the general formula

$$
E\left(M+\Delta n_{i}\right)-E(M)=\Delta n_{i} \varepsilon_{i}
$$

Summarizing the findings above, it is clear that the optimal value for $E_{0}$ is zero at the exact GKS level. (In the case of an optimized effective Kohn-Sham potential (OEP), $-E A=\varepsilon_{\text {LUMO }}+$ $\Delta_{\mathrm{XC}}{ }^{27,30}$ and thus $E_{0}=-\Delta_{\mathrm{XC}}$, where $\Delta_{\mathrm{XC}}$ is the derivative discontinuity.) Setting $E_{0}$ to zero means that only virtual orbitals with negative eigenvalues are considered, and eq 4 is reduced to

$$
E(\mathbf{r})=\frac{\sum_{i=\mathrm{LUMO}}^{\varepsilon_{i}<0} \varepsilon_{i} \rho_{i}(\mathbf{r})}{\rho(\mathbf{r})}
$$

We will hereafter refer to this quantity $E(\mathbf{r})$ as the local electron attachment energy. The choice of $E_{0}$ value has the added advantage when used together with an exact GKS-DFT method that $E(\mathbf{r})$ becomes less sensitive to the size and diffuseness of the basis set. When adding diffuse functions to a basis set, these will combine to form virtual orbitals that represent free unbound electrons. However, at the exact GKS-DFT level, these orbitals always have eigenvalues that are equal to or greater than zero even at the infinite basis set limit. Thus they will never contribute to $E(\mathbf{r})$.

In practical applications it is necessary to use an approximate GKS-DFT method. On the basis of the discussion above on Janak's theorem and the linear energy dependence upon fractional electron addition, we will argue that a standard hybrid density functional method, such as B3LYP or PBE0, that typically includes $20-25 \%$ HF exchange is a suitable choice. These methods generally provide accurate thermochemistry and kinetics for main group chemistry, including donor-acceptor interactions that involve intermolecular charge transfer. ${ }^{31}$ They are also good at reproducing experimental electron affinities. ${ }^{31}$ Furthermore, the energy dependence upon fractional electron addition is closer to linear than with the pure generalized gradient approximation (GGA) or LDA functionals. ${ }^{29}$ Another obvious choice would be a functional that includes $100 \%$ long-range $\mathrm{HF}$ exchange, such as long-range corrected GGA or double hybrid functionals. However, the orbital energies are rather sensitive to the choice of shifting parameters, and the optimum parameters are system-dependent. ${ }^{32,33}$ We have found that functionals of this type generally result in a larger energy difference between the LUMO and the LUMO+1 orbital than is obtained with the standard hybrid functionals. Our limited tests indicate that the large LUMO-LUMO+1 gaps reduce the regioselectivity information provided by $E(\mathbf{r})$. Although there is a risk that the standard hybrid functionals provide orbital energies that are slightly shifted to too low values, this is not likely to have large effects on $E(\mathbf{r})$; due to the functional form of eq 8 , the most negative virtual orbitals will dominate $E(\mathbf{r})$, and higher virtual orbitals with energies that are negative but close to zero will have small impact as long as they are few and not very localized.

Following the derivation of eq $2,{ }^{17} E(\mathbf{r})$ can be divided into different components,

$$
\begin{aligned}
& E(\mathbf{r})=\frac{1}{\rho(\mathbf{r})}\left[\sum_{i=\mathrm{LUMO}}^{\varepsilon_{i}<0} t_{i}(\mathbf{r})-V(\mathbf{r}) \sum_{i=\mathrm{LUMO}}^{\varepsilon_{i}<0} \rho_{i}(\mathbf{r})\right. \\
& \left.+V_{\mathrm{XC}}(\mathbf{r}) \sum_{i=\mathrm{LUMO}}^{\varepsilon_{i}<0} \rho_{i}(\mathbf{r})\right]
\end{aligned}
$$

where $t_{i}(\mathbf{r})$ is the local kinetic energy density of orbital $i$, herein defined as $t_{i}(\mathbf{r})=-1 / 2 \psi_{i}^{*}(\mathbf{r}) \nabla^{2} \psi_{i}(\mathbf{r})$. The $t_{i}(\mathbf{r})$ component is the only one that directly depends on the functional forms of the virtual orbitals. The electrostatic potential $[V(\mathbf{r})]$ and the KohnSham potential $\left[V_{\mathrm{XC}}(\mathbf{r})\right]$ are defined solely by the occupied orbitals and the external potential, but the scaling of their contributions is proportional to the sum of the densities of the contributing virtual orbitals. Because of the often large variations of $V(\mathbf{r})$ over a molecular isodensity surface, $V(\mathbf{r})$ has a large influence on the regioselective information provided by $E(\mathbf{r})$. The $V_{\mathrm{XC}}(\mathbf{r})$, on the contrary, is nearly constant over an isodensity surface and is thus likely to be less influential.

It should be noted that we have argued for the functional form of eq 8 based on Janak's theorem and the piecewise linear energy dependence of fractional electron addition and without invoking concepts from conceptual DFT. However, it can be shown that for some simple molecular systems that $E(\mathbf{r})$ provides similar information as the Fukui function for nucleophilic attack, $f^{+}(\mathbf{r})$, when the latter is approximated by $\rho_{\text {LUMO }}(\mathbf{r})$ within the frozen orbital approximation. In most smaller organic molecules that are nonconjugated the LUMO is the only virtual orbital with a significant negative eigenvalue. In such systems

$$
E(\mathbf{r}) \simeq \frac{2 \rho_{\mathrm{LUMO}}(\mathbf{r})}{\rho(\mathbf{r})} \varepsilon_{\mathrm{LUMO}} \simeq \frac{-2 f^{+}(\mathbf{r})}{\rho(\mathbf{r})} E A
$$

Thus $E(\mathbf{r})$ entails similar regioselectivity information as $f^{+}(\mathrm{r})$ when both are analyzed on an isodensity surface of a small molecule with positive $E A$. However, $E(\mathbf{r})$ may be better than $f^{+}(r)$ alone for quantifying the difference in global reactivity between systems due to the multiplication of $f^{+}(\mathrm{r})$ by $E A$ in eq 10. In addition, $E(\mathbf{r})$ is likely to be more versatile than $f^{+}(r)$ in describing the reactivity of complex systems. The latter approach has problems describing systems with degenerate or neardegenerate LUMO and LUMO+1 orbitals. As exemplified in Figure 1 , the reactivity of a system with two $\pi$ regions $\left(\mathbf{2}_{n}\right)$ separated by a $\sigma$ region cannot be compared directly to that of a single $\pi$-region system (1). When the length of the $\sigma$ region increases ( $n$ increases), the reactivity of each $\pi$ region in $2_{n}$ is expected to converge to the reactivity of the $\pi$ region in $\mathbf{1}$. As

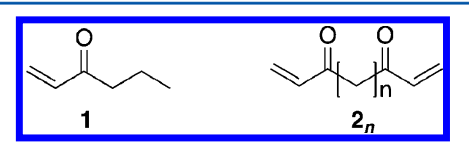

Figure 1. Two systems of different size but with similar reactivity of the $\pi$ regions at large $n$. The surface $E(\mathbf{r})$ at the $\pi$ region $(\mathrm{C}=\mathrm{C}-\mathrm{C}=\mathrm{O})$ of $\mathbf{1}$ differs by $<0.04 \mathrm{eV}$ from the corresponding regions in $\boldsymbol{2}_{n}$ already when $n$ $=4$, whereas $f^{+}(\mathbf{r})$ of $2_{n}$ converges to $0.5 f^{+}(\mathbf{r})$ of $\mathbf{1}$. Details of the sizeconsistency analysis can be found in Table S1 in the Supporting Information. 


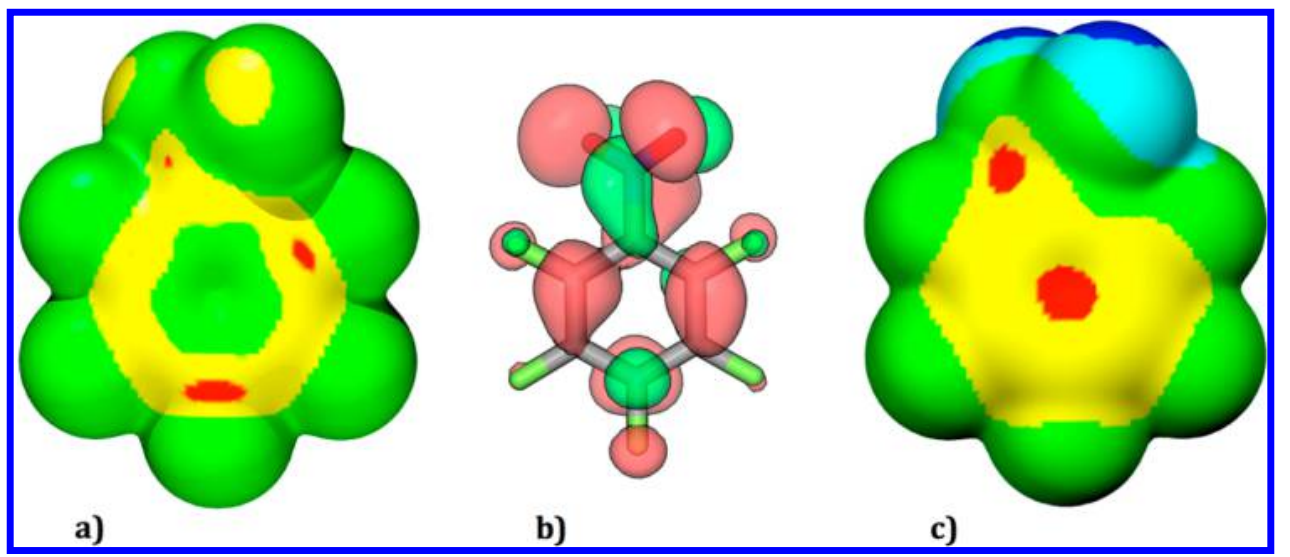

Figure 2. (a) Computed $E_{\mathrm{S}}(\mathbf{r})$ of pentafluoronitrobenzene $\left(\mathrm{C}_{6} \mathrm{~F}_{5} \mathrm{NO}_{2}\right)$ at the 0.004 au isodensity surface. Color ranges for $E_{\mathrm{S}}(\mathbf{r})$ in eV: red $<-2.05$, $-2.05<$ yellow $<-1.10$, and green $>-1.10$. (b) LUMO of $\mathrm{C}_{6} \mathrm{~F}_{5} \mathrm{NO}_{2}$. (c) Computed $V_{\mathrm{S}}(\mathbf{r})$ of $\mathrm{C}_{6} \mathrm{~F}_{5} \mathrm{NO}_{2}$ at the 0.001 au isodensity surface. Color ranges for $V_{S}(\mathbf{r})$ in $\mathrm{kcal} / \mathrm{mol}$ : red $>25,15<$ yellow $<25,-10<$ green $<15,-20<$ cyan $<-10$, and blue $<-20$.

shown in Table S1 in the Supporting Information, $E(\mathbf{r})$ correctly predicts this behavior, whereas $f^{+}(\mathbf{r})$ of $\mathbf{2}_{n}$ converges to $0.5 f^{+}(\mathbf{r})$ of 1. It can be argued that $f^{+}(\mathbf{r})$ should be renormalized for large $n$ when LUMO and LUMO+1 are close to degenerate. However, this will not solve the size-consistency problem entirely, as $f^{+}(\mathbf{r})$ will make a discontinuous jump at the $n$ for which the energy difference between the two orbitals becomes below the defined tolerance.

It is also known that frontier orbital descriptions do not always provide a complete reactivity picture in systems, such as aromatic molecules, where there are several $\pi$ - or $\pi^{*}$-orbitals close in energy. This has been recognized by Langenaeker et al. ${ }^{34}$ and Andersson et al. ${ }^{35}$ Since $\bar{I}_{S}(\mathbf{r})$, which use a similar orbital expansion as $E(\mathbf{r})$, is more effective than the $\rho_{\text {HOMO }}(\mathbf{r})$ for electrophilic attack on aromatic systems, ${ }^{20}$ we anticipate that $E(\mathbf{r})$ may be more successful than $\rho_{L U M O}(\mathbf{r})$ for nucleophilic attack in aromatic systems. The validity of this assumption has been investigated in the current study. There are also potential advantages of $E(\mathbf{r})$ compared to a frontier orbital description in systems where there are a both low-lying $\sigma^{*}$ and $\pi^{*}$ orbitals. In such a system, the LUMO has to be of either type, and cannot describe the reactivity of the other. It can be expected that $E(\mathbf{r})$ should be able to predict reactivity also in very large systems with multiple interaction sites, like a protein, or systems with near infinite size and a band structure, like a semiconductor. We even see the potential to use $E(\mathbf{r})$ in connection with periodic boundary conditions to treat infinite systems.

In the following study, we have analyzed the potential of $E(\mathbf{r})$ computed on an isodensity surface $\left[E_{S}(\mathbf{r})\right]$ to predict the propensity for nucleophilic attack in a variety of systems and in relation to different types of reactions and intermolecular interactions. To evaluate the predictive capacity of $E_{S}(\mathbf{r})$, we will compare the positional selectivity obtained by $E_{\mathrm{S}}(\mathbf{r})$ to that of $\rho_{\text {LUMO }}(\mathbf{r})$ and the global reactivity to the $\varepsilon_{\text {LUMO }}$ and Parr's electrophilicity index, $\omega .^{36}$ The latter is defined by

$$
\omega=\frac{\mu^{2}}{\eta}
$$

where $\mu$ is the electronic chemical potential and $\eta$ is the absolute hardness, and they are given by $\mu=\left(\varepsilon_{\mathrm{LUMO}}+\varepsilon_{\mathrm{HOMO}}\right) / 2$ and $\eta=$ $\left(\varepsilon_{\mathrm{LUMO}}-\varepsilon_{\mathrm{HOMO}}\right) / 2$ in the frozen orbital approximation. ${ }^{36}$ The reason for the comparison with $\omega$ is not to determine which is the better electrophilicity descriptor but rather to investigate the effects of going beyond a frontier orbital description, and thereby justify the introduction of $E_{S}(\mathbf{r})$ as a complementary tool for analysis and prediction of interactions with nucleophiles.

\section{COMPUTATIONAL METHODS}

Molecular geometries were optimized and characterized by harmonic frequency analysis at the B3LYP/6-31G* level. Singlepoint calculations were performed to obtain the occupied and virtual orbitals at the B3LYP/6-31+G(d,p) level. This level of theory generally provides virtual orbitals that work well together with eq 8. All KS-DFT computations were performed using the Gaussian 09 suite of programs. ${ }^{37}$

$E_{\mathrm{S}}(\mathbf{r})$ and $V_{\mathrm{S}}(\mathbf{r})$ were computed on molecular isodensity surfaces using a modified version of the HS95 program developed by one of the authors (T. Brinck). In previous applications of the surface property approach, it has been common to compute $V_{S}(\mathbf{r})$ or $\bar{I}_{S}(\mathbf{r})$ at molecular surfaces defined by the 0.002 or 0.001 au contours of the electron density. These surfaces encompass $95-98 \%$ of a molecules electron density and typically lie outside of the van der Waals radii of the constituting atoms. ${ }^{8}$ However, in using $E_{S}(\mathbf{r})$, we have found generally better agreements with experimental measures of local reactivity if a slightly higher density value, that is, $0.004 \mathrm{au}$, is used to define the surface. A possible explanation for this result is that $E_{\mathrm{S}}(\mathbf{r})$ in most instances has been used to model interactions that are stronger and more short-ranged than van der Waals interactions. Graphical visualization of surface properties and orbital densities has been performed using the UCSF Chimera package. ${ }^{38}$ The positions and magnitudes of local surface minima have been determined using a numerical procedure implemented in the HS95 program. The results are in agreement with those obtained using an alternative procedure by Bulat et al. that is implemented in the WFA-program. ${ }^{8}$

Linear regression has been used to analyze correlations between computed descriptors and experimental rate constants or computed interaction energies. The statistical significance of the correlations has been analyzed in terms of correlation coefficients $\left(R^{2}\right)$ and standard errors (SEs). We have also computed the cross-validated correlation coefficient $\left(Q^{2}\right)$ by means of the leave-one-out procedure. ${ }^{39}$ A detailed account of the results from the statistical analysis is found in Supporting Information. 


\section{RESULTS AND DISCUSSION}

Nucleophilic Aromatic Substitution. Figure 2 a shows $E_{S}(\mathbf{r})$ of pentafluoronitro-benzene. The regions of lowest $E_{S}(\mathbf{r})$ are found over the $\pi$-bonds, where also the $\pi^{*}$-densities are expected to be highest. The lowest $E_{\mathrm{S} \text {,min }}$ are located over ortho and para positions, and their values are $-2.11 \mathrm{eV}$ and $-2.21 \mathrm{eV}$, respectively. Due to the $C_{2}$-symmetry of the molecule, there is also a second unique $E_{\mathrm{S} \text {,min }}$ at the ortho positions, with a value of $-2.00 \mathrm{eV}$. The $E_{\mathrm{S}, \min }$ over the meta positions are significantly higher, -1.88 and $-1.83 \mathrm{eV}$. These results give a picture that is consistent with the experimentally observed ortho-para positional selectivity of perfluoronitrobenzene in nucleophilic aromatic substitution $\left(S_{\mathrm{N}} \mathrm{Ar}\right)$ reactions. ${ }^{40}$ There is also a relatively low $E_{\mathrm{S}, \min }(-2.06 \mathrm{eV})$ over the nitrogen, indicating this to be another potential site for nucleophilic attack. Much higher $E_{\mathrm{S} \text {,min }}$ are found over the oxygens and fluorines. Their values are in the ranges -1.64 to -1.65 and -0.30 to $-0.36 \mathrm{eV}$, respectively. The latter are indicative of the fluorine $\sigma^{*}$ orbitals.

Four virtual $\pi^{*}$ orbitals with negative eigenvalues of -3.50 , $-1.84,-1.78$, and $-1.64 \mathrm{eV}$ contribute to $E_{\mathrm{S}}(\mathbf{r})$. This gives a good representation of the electron accepting tendencies and the susceptibility for nucleophilic attack in this molecule. It can be noted that an analysis of the LUMO, as shown in Figure $2 b$, provides less information. The LUMO has lobes over the $\mathrm{C}-\mathrm{N}$ bond, the nitro oxygens, the ortho-meta $\mathrm{C}-\mathrm{C}$ bond, and the para carbon with sizes that decrease in the same order. Thus, the ortho-para $S_{N} A r$ positional selectivity is less obvious from the LUMO. Also, the $V_{S}(\mathbf{r})$, shown in Figure $2 c$, gives a very different picture of the nucleophilic reactivity than $E_{S}(\mathbf{r})$. Here the most positive $V_{S}(\mathbf{r})$ regions are over the $\mathrm{C}-\mathrm{N}$ bond and in the center of the aromatic ring.

The good agreement between the $S_{N} A r$ regioselectivity and the magnitude of the $E_{S \text {,min }}$ prompted us to investigate whether the $E_{\mathrm{S} \text {,min }}$ correlates with the relative $S_{\mathrm{N}} \mathrm{Ar}$ reactivities of congeners of aromatic substrates. In Table 1, we list the $E_{\mathrm{S} \text {,min }}$ and experimental rate constants $(k)^{41,42}$ for the amination of nine fluorine-substituted heteroaromatics, mostly pyridines (see Figure 3 for structures). In all cases, the lowest $E_{\mathrm{S}, \min }$ is found over the carbon that corresponds to the positionally preferred site for $S_{N} A r$. For two of the substrates, there is a product distribution between two isomers, and the individual rate constants for forming the isomers have been determined. In these cases, the second lowest $E_{\mathrm{S}, \min }$ is found at the position leading to the minor isomer.

There is a linear correlation between the experimental $\ln k$ values and $E_{\mathrm{S}, \min }$ with a coefficient of determination $\left(R^{2}\right)$ of 0.81 , as shown in Figure 3. The $R^{2}$ value improves to 0.86 if only the reactivity at the most favored reaction site (highest $\ln k$ ) of each system is considered. The correlations clearly indicate that $E_{\mathrm{S}}(\mathbf{r})$ reflects the susceptibility for $S_{N} A r$ in these systems. The correlations are not as good as those that have been obtained between $\ln k$ and $\bar{I}_{S \text {,min }}$ for electrophilic aromatic substitution $\left(S_{E} A r\right) .{ }^{20}$ This is not surprising considering that this type of $S_{N} A r$ reactions have a late transition state that is close in structure to the product, that is, the sigma-complex, of the rate-determining step. ${ }^{43}$ In contrast, $S_{\mathrm{E}} \mathrm{Ar}$ reactions, and particularly nitrations, have an early transition state that resembles the reaction complex in structure. ${ }^{44}$ We have also shown that the computed relative sigma-complex (Meisenheimer complex) energies of $\mathrm{S}_{\mathrm{N}} \mathrm{Ar}$ with fluorine as nucleofuge correlate very well with the relative rate constants. ${ }^{43}$ However, this requires proper consideration of solvation effects to obtain stable sigma complexes. Thus, in this
Table 1. Minima in $E_{S}(r)\left(E_{S, \min }\right)$ and Logarithm of the Experimental Rate Constants $\left(\ln k\right.$ ) for $S_{N} A r$ at the Corresponding Carbon Positions for the Molecules of Figure $3^{a, b}$

$\begin{array}{cccc}\text { structure } & \text { position }^{c} & E_{S, \text { min }}(\mathrm{eV}) & \ln k \\ \mathrm{a} & 2 & -1.51 & -13.38 \\ & 3 & -1.43 & (-)^{d} \\ \mathrm{~b} & 2 & -1.50 & -12.04 \\ & 6 & -1.20 & (-)^{d} \\ \mathrm{c} & \mathrm{eq} & -1.82 & -9.89 \\ \mathrm{~d} & 2 & -1.63 & (-)^{d} \\ & 4 & -2.27 & -7.29 \\ \mathrm{e} & 2 & -1.57 & (-)^{d} \\ & 4 & -2.11 & -6.26 \\ & 6 & -1.68 & (-)^{d} \\ \mathrm{f} & 4 & -2.57 & -3.68 \\ \mathrm{~g} & 2 & -1.94 & (-)^{d} \\ & 4 & -2.53 & -2.94 \\ & 6 & -2.10 & -3.63 \\ \mathrm{~h} & 2 & -2.41 & 0.27 \\ & 4 & -2.73 & 1.22 \\ \mathrm{I} & 2 & -1.92 & (-)^{d} \\ & 4 & -2.37 & 0.30 \\ \boldsymbol{R}^{2} & & & 0.81\end{array}$

${ }^{a} E_{S}(\mathbf{r})$ was computed at the 0.004 au density surface. ${ }^{b}$ Experimental rate constants (in $\mathrm{L} \mathrm{mol}^{-1} \mathrm{~s}^{-1}$ ) were obtained with $\mathrm{NH}_{3}$ as nucleophile in $60 \%$ dioxane $-40 \%$ water at $25{ }^{\circ} \mathrm{C}$ and are taken from refs 41 and 42. ${ }^{c}$ Only positions with an $E_{\mathrm{S}, \min }$ are listed. ${ }^{d}$ No product was observed.

context, it is remarkable that an approach, such as $E_{\mathrm{S}}(\mathbf{r})$, that is based solely on characterization of the isolated reactant in the gas phase, can provide estimates of relative reactivity and positional selectivity. For comparison, it can be noted that there is no significant correlation between $\ln k$ of the favored reaction and $\omega$ for this reaction series, $R^{2}=0.12$. The correlation between $\ln k$ and $\varepsilon_{\mathrm{LUMO}}$ is of similar quality, $R^{2}=0.11$. We are expecting that $E_{S}(\mathbf{r})$ will work even better for predicting reactivities of $S_{N} A r$ with chlorine or bromine as nucleofuges, since we have found these reactions to be concerted with an early transition state that resembles the reactant in structure. ${ }^{45}$ This will be a focus of a future study.

Nucleophilic Addition to Activated Double Bonds. Figure 4 shows $E_{S}(\mathbf{r})$ of benzylidenemalonitrile. The lowest $E_{S, \min }$ over the aromatic ring is again found at the ortho and para positions, consistent with the positional selectivity for $S_{N} A r$ associated with the resonance-withdrawing substituent. However, the overall lowest $E_{\mathrm{S}, \min }$ is at the $\beta$-carbon. This is also the prevalent site for interaction with strong nucleophiles in this type of molecules. Bernasconi and Killion have investigated the mechanism and kinetics for conjugate addition of piperidine to a series of substituted benzylidenemalonitriles in a 50\% DMSO$50 \%$ water solution. ${ }^{46}$ The initial step in these reactions is the formation of a zwitterionic intermediate that has the piperidine nitrogen bonded to the $\beta$-carbon. In Figure 5 the $\ln k$ for this initial process is plotted against the $E_{\mathrm{S} \text {,min }}$ on the $\beta$-carbon for the benzylidenemalonitriles. There is a good linear correlation between $\ln k$ and $E_{\mathrm{S} \text {,min }}$ with $R^{2}=0.92$. Thus in this series $E_{\mathrm{S}}(\mathbf{r})$ is able to quantitatively reproduce the observed reactivity with relatively high accuracy. It can be noted that $E_{\mathrm{S} \text {,min }}$ significantly overestimates the reactivity of the $4-\mathrm{NMe}_{2}$ substituted compound; the $R^{2}$ value improves to 0.98 when it is excluded 


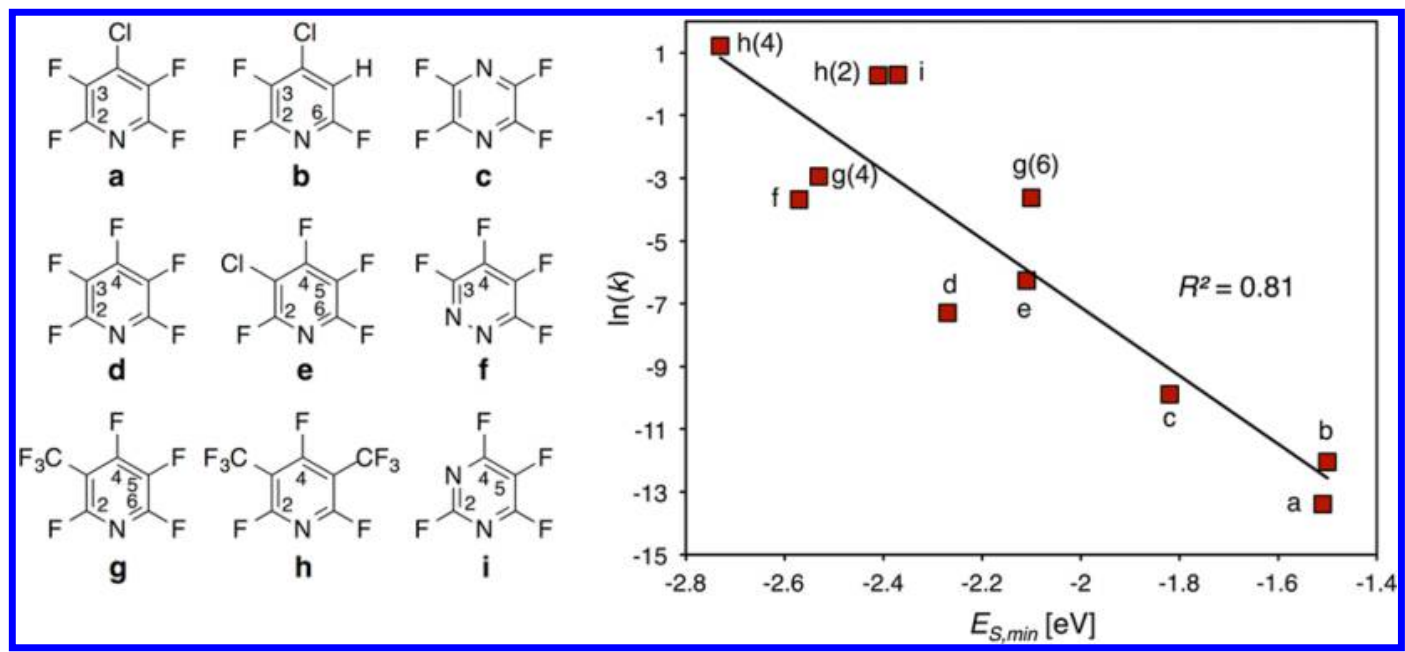

Figure 3. Structures of the heteroaromatic molecules investigated in relation to their $S_{N} A r$ reactivity and the numbering of their positions (to the left). Plot of the logarithm of the experimental rate constant $(\ln k)$ versus minimum in $E_{\mathrm{S}}(\mathbf{r})\left(E_{\mathrm{S} \text {, min }}\right)$ at the corresponding carbon position (to the right). Data from Table 1 .

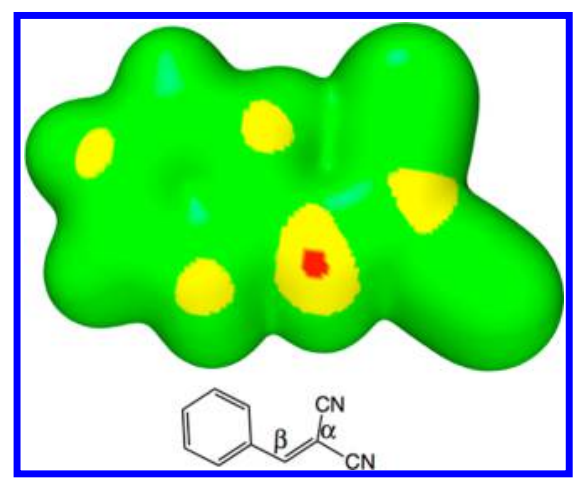

Figure 4. Computed $E_{\mathrm{S}}(\mathbf{r})$ of benzylidenemalononitrile at the 0.004 au isodensity surface. Color ranges for $E_{\mathrm{S}}(\mathbf{r})$ in $\mathrm{eV}$ : red $<-2.6,-2.6<$ yellow $<-0.8$, and green $>-0.8$. The lowest $E_{\mathrm{S}, \min }(-2.79 \mathrm{eV})$ is found at the reactive $\beta$ position. Molecular structure is shown below surface.

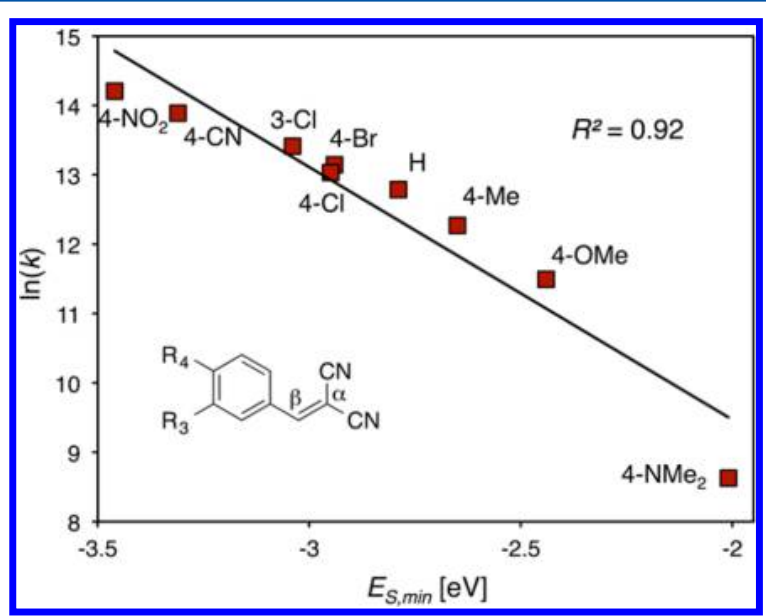

Figure 5. Plot of the logarithm of the experimental rate constant $(\ln k)$ versus $\mathrm{E}_{\mathrm{S}, \min }$ at the $\beta$ position for a series of benzylidenemalononitriles. The rate constants (in $\mathrm{L} \mathrm{mol}^{-1} \mathrm{~s}^{-1}$ ) are for the conjugate addition of piperidine in 50\% DMSO-50\% water and are taken from ref 46

from the correlation. Domingo et al. ${ }^{47}$ analyzed the reactivity of the same series using $\omega$. They also found a linear relationship with $\ln k$, but the quality of the correlation is lower and $R^{2}$ is only 0.75. Interestingly, also in their correlation $4-\mathrm{NMe}_{2}$ is an outlier and $R^{2}$ improves to 0.90 when the compound is excluded. Domingo et al. suggest that the deviation of $4-\mathrm{NMe}_{2}$ can be attributed to solvation effects.

To elucidate whether $E_{S}(\mathbf{r})$ can reproduce the reactivity of activated double bonds toward anionic nucleophiles, we computed $E_{S}(\mathbf{r})$ on a series of substituted $\alpha$-nitrostilbenes. The kinetics for addition of $\mathrm{HOCH}_{2} \mathrm{CH}_{2} \mathrm{~S}^{-}$to the $\beta$-carbon of the stilbenes in 50\% DMSO-50\% water solution has been determined by Bernasconi and Killion. ${ }^{48}$ Also, for this series, we find that the lowest $E_{S, \min }$ is found at the $\beta$-carbon. Furthermore, there is an excellent linear correlation between $\ln$ $k$ and $E_{\mathrm{S} \text {,min }}$ with an $R^{2}$ of 0.986 (Figure 6). This confirms that

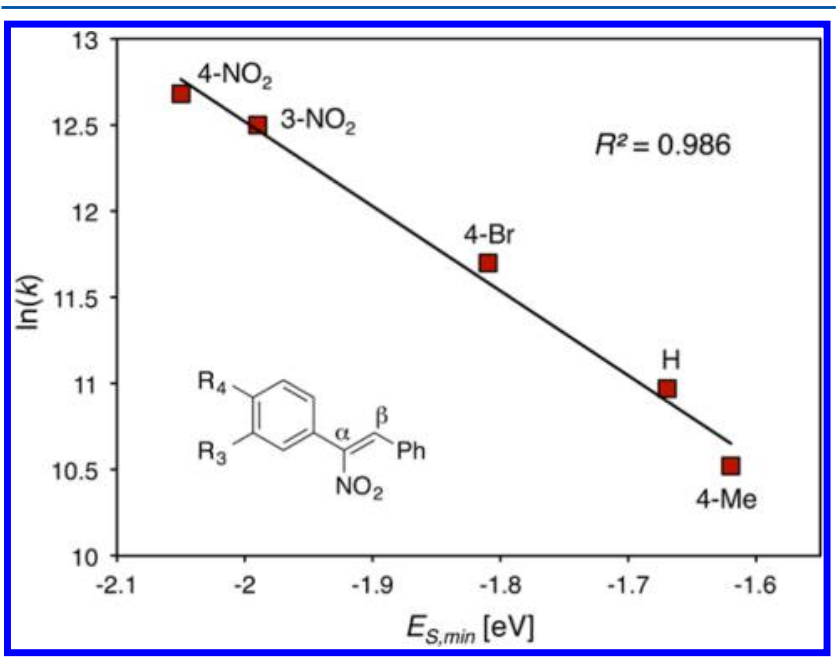

Figure 6. Plot of the logarithm of the experimental rate constant $(\ln k)$ versus $E_{\mathrm{S}, \min }$ at the $\beta$ position for a series of $\alpha$-nitrostilbenes. The rate constants (in $\mathrm{L} \mathrm{mol} \mathrm{m}^{-1} \mathrm{~s}^{-1}$ ) are for the conjugate addition of $\mathrm{HOCH}_{2} \mathrm{CH}_{2} \mathrm{~S}_{2}{ }^{-}$in $50 \%$ DMSO-50\% water and are taken from ref 48 .

$E_{S}(\mathbf{r})$ has a capacity for quantitatively ranking substrates for conjugative nucleophilic addition also when the nucleophile is anionic. It can be noted that $\omega$ performs better for the $\alpha$ nitrostilbenes than for the previous series. ${ }^{47}$ The $R^{2}$ is 0.95 and thus the correlation again is less than that between $\ln k$ and $E_{\mathrm{S} \text {,min }}$.

Halogen Bonding. Figure 7 shows $E_{S}(\mathbf{r})$ of methyl bromide. There is an $E_{S, \min }$ of $-1.14 \mathrm{eV}$ on the methyl group at the 


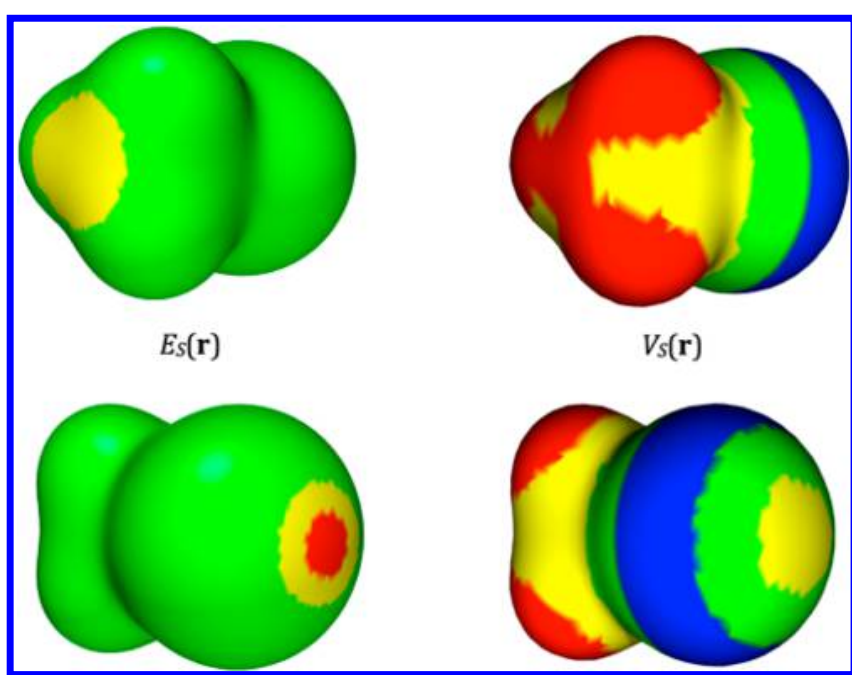

Figure 7. Computed $E_{\mathrm{S}}(\mathbf{r})$ (left) and $V_{\mathrm{S}}(\mathbf{r})$ (right) at the $0.004 \mathrm{au}$ isodensity surface of methyl bromide. The top and bottom views have the methyl group and the bromine, respectively, pointing toward the viewer. Color ranges for $E_{S}(r)$ in $\mathrm{eV}$ : red $<-1.2,-1.2<$ yellow $<-0.8$, and green $>-0.8$. Color ranges for $V_{\mathrm{S}}(\mathbf{r})$ in $\mathrm{kcal} / \mathrm{mol}$ : red $>45,10<$ yellow $<45,-10<$ green $<10$, and blue $<-20$.

intersection of the three hydrogens, corresponding to the position for nucleophilic attack in an $S_{\mathrm{N}} 2$ reaction. The $E_{\mathrm{S} \text {,min }}$ becomes more negative in the order of $\mathrm{CH}_{3} \mathrm{Cl}, \mathrm{CH}_{3} \mathrm{Br}, \mathrm{CH}_{3} \mathrm{I}$, in agreement with increasing leaving group strength for $S_{\mathrm{N}} 2$. However, the lowest $E_{\mathrm{S} \text {,min }}(-1.35 \mathrm{eV})$ of $\mathrm{CH}_{3} \mathrm{Br}$ is not found at the methyl group but at the tip of the bromine opposite to the bromine-carbon bond. A similar picture is given by the $V_{S}(\mathbf{r})$, which has a maximum $\left(V_{\mathrm{S}, \max }\right)$ at the tip of the bromine with a magnitude of $25.7 \mathrm{kcal} / \mathrm{mol}$. However, in the $V_{\mathrm{S}}(\mathbf{r})$, the most positive areas, with $V_{\mathrm{S}, \max }$ of $38.2 \mathrm{kcal} / \mathrm{mol}$, are found at the methyl hydrogens. These high positive values reflect the hydrogen-bond-donating properties of the molecule. Positive $V_{S, \max }$ on the halogens has been associated with noncovalent interactions with nucleophiles in what today is referred to as halogen bonding. ${ }^{10}$ Furthermore, the halogen bond strength and positional selectivity often correlate with the halogen $V_{\mathrm{S} \text {, max }}$ in congeneric series of halogenated compounds. ${ }^{13,15,49}$ However, when comparing $E_{S}(\mathbf{r})$ with $V_{S}(\mathbf{r})$, it is clear that the former to a greater extent emphasizes the electrophilic nature of the bromine. This prompted us to investigate whether there is a correlation between halogen bond strength and $E_{\mathrm{S}, \min }$. The $E_{\mathrm{S}}(\mathbf{r})$ was computed for a series of methyl chlorides and methyl bromides, with varying degree of hydrogen-fluorine substitution, previously studied by Riley and Hobza. ${ }^{50}$ The halogen bond interaction energies $\left(\Delta E_{\text {int }}\right)$ for complexes between these compounds and formaldehyde were computed at the CCSD(T)/aug-cc-pVTZ level. ${ }^{50}$ There is indeed a very good linear correlation between $\Delta E_{\text {int }}$ and $E_{S, \text { min }}$ with an $R^{2}$ value 0.970 and an SE of only $0.09 \mathrm{kcal} / \mathrm{mol}$ (Figure 8). The corresponding correlation between $\Delta E_{\text {int }}$ and $V_{\mathrm{S} \text {, max }}$ is of slightly lower quality $\left.\left(R^{2}=0.952, \mathrm{SE}=0.12 \mathrm{kcal} / \mathrm{mol}\right)\right)$.

The good correlation between $\Delta E_{\text {int }}$ and $E_{\mathrm{S} \text {,min }}$ could be taken as an indication that charge transfer is of significant importance for halogen bonding. This would be in accordance with the early interpretation of halogen bonding as a donor-acceptor interaction; electron density from the donor is transferred to the antibonding $\sigma^{*}$ orbital of the halogen. In recent years, there has instead been an emphasis on the electrostatic nature of the halogen bond, and electrostatics together with dispersion have

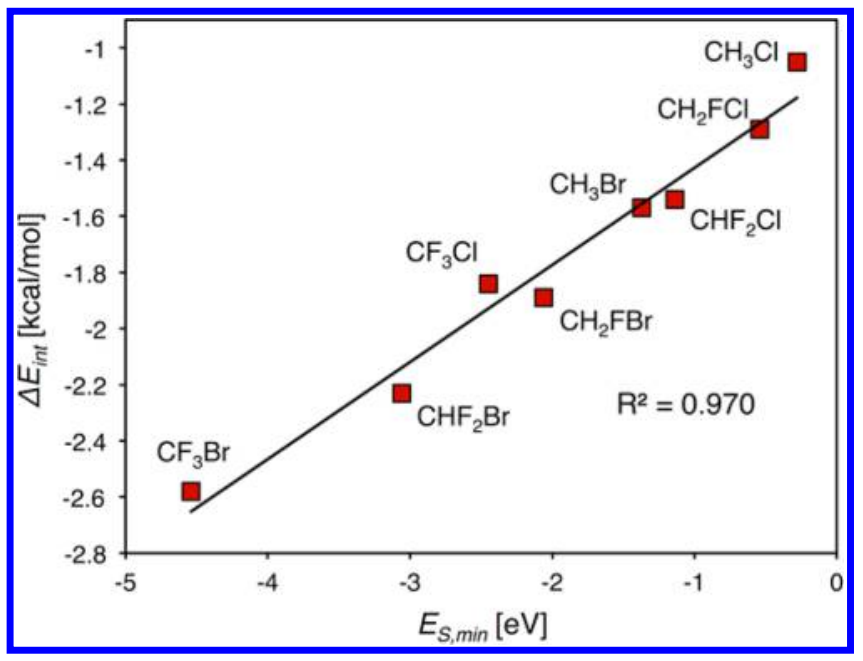

Figure 8. Plot of the halogen bond interaction energy versus the halogen $E_{\mathrm{S} \text {,min }}$ for a series of halomethanes. Interaction energies are for the interaction with formaldehyde, computed at the $\operatorname{CCSD}(\mathrm{T}) /$ aug-ccpVTZ level, and taken from ref 50 . The $E_{S, \min }$ is consistently located at the tip of the chlorine or bromine.

been indicated to govern the strengths of halogen bonds. ${ }^{14,51,52}$ The polarization of the $\sigma$ orbital toward the halogen-carbon bond results in depletion of electron density outside of the halogen atom opposite to the bond, that is, a so-called $\sigma$ hole is formed where the electrostatic potential is positive. ${ }^{51}$ The location of the $\sigma$ hole largely coincides with the region where the $\sigma^{*}$ orbital has its highest density. Thus it is logical that the halogen $V_{\mathrm{S}, \max }$ and the $E_{\mathrm{S} \text {,min }}$ are found at the same position. It should also be realized that $E(\mathbf{r})$ according to eq 9 has a significant contribution from $V(\mathbf{r})$, and this could explain the linear correlation between the halogen $V_{\mathrm{S}, \max }$ and $E_{\mathrm{S}, \min }\left(R^{2}=\right.$ $0.95)$.

In the case of the halogenated methanes, the LUMO is always a $\sigma^{*}$ orbital associated with the heaviest halogen. This is also reflected by the good correlation between $\Delta E_{\text {int }}$ and $\varepsilon_{\text {LUMO }}, R^{2}=$ 0.968 . Also, $\omega$ correlates well with $\Delta E_{\text {int }}\left(R^{2}=0.94\right)$, which can be traced to the contribution of $\varepsilon_{\text {LUMO }}$ to $\omega$.

To determine if $E_{S}(\mathbf{r})$ can describe the halogen bonding of more complex systems with multiple interaction sites for nucleophilic attack, we have studied a series of halogenated benzenes, of the type $\mathrm{C}_{6} \mathrm{H}_{(5-y)} \mathrm{F}_{y} X$, where $y=0,2,5$ and $X=\mathrm{Cl}$, $\mathrm{Br}$, I. This series poses an interesting challenge for $E_{S}(\mathbf{r})$ since the molecules generally have a multitude of negative virtual orbitals and the LUMO is for half of the molecules an aromatic $\pi^{*}$ orbital and for the other half a halogen $\sigma^{*}$ orbital; $\mathrm{C}_{6} \mathrm{~F}_{5} \mathrm{Cl}, \mathrm{C}_{6} \mathrm{~F}_{5} \mathrm{Br}$, and the iodobenzenes are the molecules of the latter type.

Figure 9 shows the $E_{\mathrm{S}}(\mathbf{r})$ of 1-bromo-3,5-difluorobenzene, and there are regions of low $E_{S}(\mathbf{r})$ associated with both the aromatic ring and the $\mathrm{Br}$. Interestingly, the lowest $E_{\mathrm{S}, \min }$ is found at the tip of the heavy halogen despite the fact that the LUMO is an aromatic $\pi^{*}$ orbital; a feature that 1-bromo-3,5-difluorobenzene shares with 1-bromo-2,4-difluorobenzene and bromobenzene. There are also higher $E_{\mathrm{S}, \min }$ associated with the different aromatic carbons that reflect their varying susceptibility for nucleophilic attack.

Riley et al. have investigated the halogen bonding of the molecules of this series and computed interaction energies for complexes with acetone at the MP2/aug-cc-pVDZ(-PP) level. ${ }^{49}$ We have considered the interaction energies for the perpendicular approach toward the heavy halogen $(\mathrm{X})$, that is, 


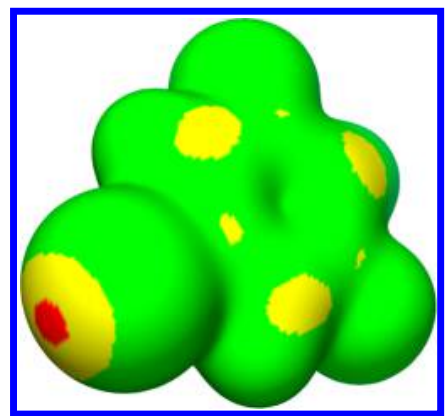

Figure 9. Computed $E_{S}(\mathbf{r})$ of 1-bromo-3,5-difluorobenzene at the 0.004 au isodensity surface. Color ranges for $E_{\mathrm{S}}(\mathbf{r})$ in $\mathrm{eV}$ : red $<-2.0,-2.0<$ yellow $<-0.8$, and green $>-0.8$. The lowest $E_{\mathrm{S}, \min }(-2.27 \mathrm{eV})$ is found at the tip of the bromine.

constrained complexes with a $\mathrm{X}-\mathrm{O}-\mathrm{C}$ angle of $180^{\circ}$, because the fully relaxed structures, in addition to the halogen bond, involve interactions between acetone and the ortho-substituents ( $\mathrm{F}$ or $\mathrm{H})$ of the halobenzenes.

Also, for this series there is a very good linear correlation between $\Delta E_{\text {int }}$ and the halogen $E_{\mathrm{S} \text {,min }}$ with an $R^{2}=0.979$ and $\mathrm{SE}=$ $0.18 \mathrm{kcal} / \mathrm{mol}$ (Figure 10). This is remarkable considering that the donating halogen varies between $\mathrm{Cl}, \mathrm{Br}$, and $\mathrm{I}$. Not surprisingly, the correlations with $\varepsilon_{\text {LUMO }}$ and $\omega$ are of much lower quality, $R^{2}=0.82$ and 0.77 , respectively. As expected, and in agreement with the work of Riley et al., ${ }^{49}$ we find a good correlation between $\Delta E_{\text {int }}$ and the halogen $V_{S, \max } R^{2}=0.975$ and $\mathrm{SE}=0.19 \mathrm{kcal} / \mathrm{mol}$. Despite the fact that both $E_{\mathrm{S}, \min }$ and $V_{\mathrm{S}, \max }$ correlate well with $\Delta E_{\text {int }}$ and with similar $R^{2}$, the linear correlation between $E_{\mathrm{S}, \min }$ and $V_{\mathrm{S} \text {, max }}$ is less, $R^{2}=0.93$. Our analysis indicates that $E_{\mathrm{S}, \min }$ and $V_{\mathrm{S} \text {,max }}$ provide complementary information for describing the halogen bond strength in this system. An excellent correlation for $\Delta E_{\text {int }}$ is obtained when $E_{\mathrm{S}, \min }$ and $V_{S, \max }$ are used as independent variables in a multiple linear relationship, $R^{2}=0.993$ and $S E=0.10 \mathrm{kcal} / \mathrm{mol}$. The crossvalidated correlation coefficient $Q^{2}$ is as high as 0.990 , clearly indicating that the excellent correlation is not the result of overfitting. It is also obvious from Figure 10 that there is a consistent improvement in the correlation going from the onevariable to the two-variable relationship.

It is important to remember that there is no unique method for decomposing the interaction energy of an intermolecular interaction, and this is one reason behind the conflicting views on the importance of electrostatics versus charge transfer for halogen bonding that can be found in current literature. ${ }^{14,52-56}$ Furthermore, in the classical analysis of donor-acceptor interactions, the charge-transfer interaction energy $\left(\Delta E_{\mathrm{CT}}\right)$ includes terms that in most energy decomposition analyses are considered electrostatic. Reed et al. recognized this already in their review of donor-acceptor interactions when they compared $\Delta E_{\mathrm{CT}}$ obtained from natural bond orbitals (NBO) to Morokuma energy decomposition analysis. ${ }^{57}$ An analogy can be made to $E(\mathbf{r})$, which has a contribution from the electrostatic potential, $V(\mathbf{r})$, according to eq 9. Still, independent of the definition of charge transfer, it is a limitation to only consider the importance of electrostatics when describing halogen bonding. In a recent study by Rosokha et al., much higher and more general correlations were found when the halogen bond interaction energy was correlated by a multilinear relationships with $V_{S \text {, max }}$ and $\Delta E_{\mathrm{CT}}$ (obtained by NBO) than when the same descriptors where used alone. ${ }^{53}$ Our results for the halogenated benzenes indicate that the halogen $V_{\mathrm{S}, \max }$ and $E_{\mathrm{S}, \min }$, in a similar

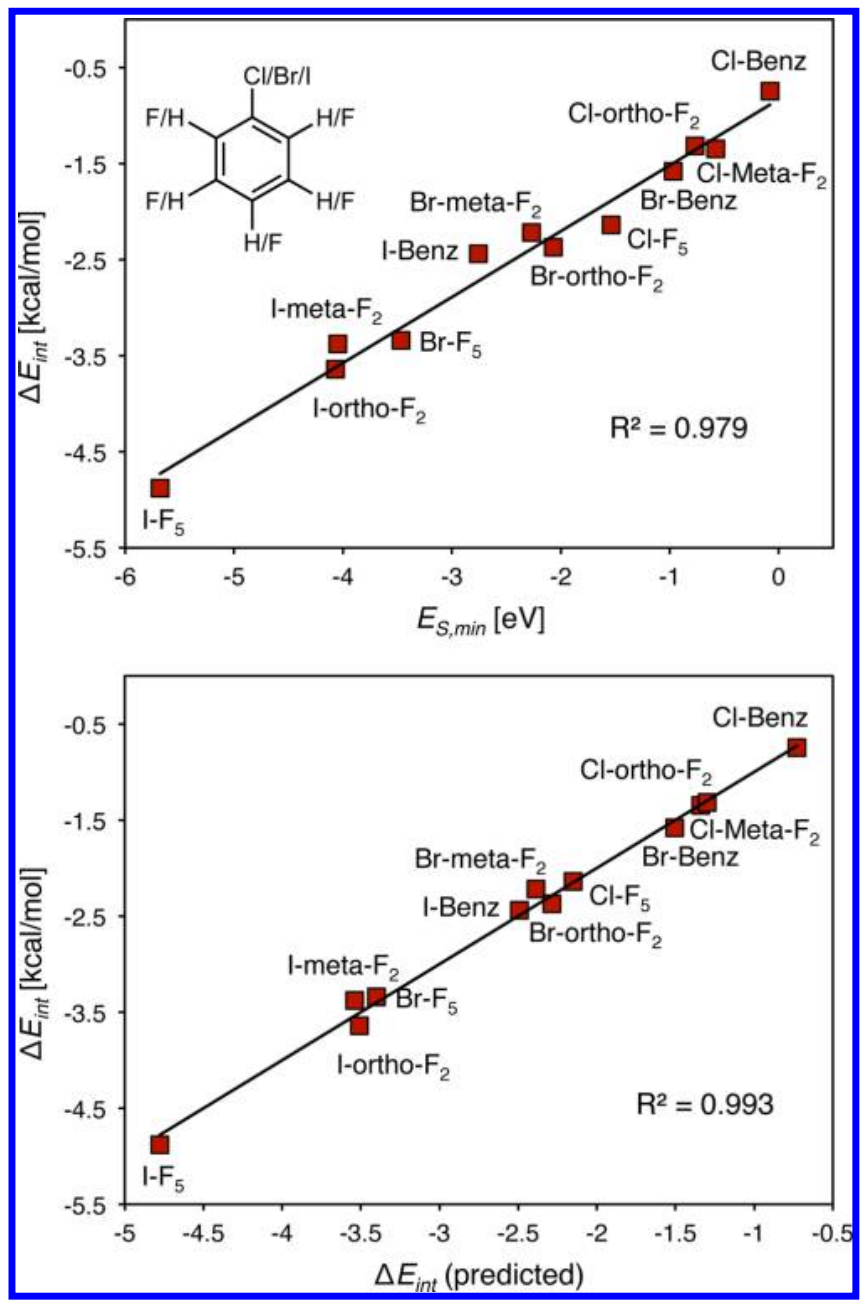

Figure 10. Correlations of the halogen bond interaction energy $\left(\Delta E_{\text {int }}\right)$ for a series of halobenzenes of the type $\mathrm{C}_{6} \mathrm{H}_{(5-y)} \mathrm{F}_{y} \mathrm{X}$, where $\mathrm{X}=\mathrm{Cl}, \mathrm{Br}$, I. $\Delta E_{\text {int }}$ are for the interaction with acetone, computed at the MP2/aug-cc$\mathrm{pVDZ}(-\mathrm{PP})$ level and taken from ref 49. The top plot shows $\Delta E_{\text {int }}$ versus the halogen $(\mathrm{Cl} / \mathrm{Br} / \mathrm{I}) E_{\mathrm{S}, \text { min }}$. The lower plot shows $\Delta E_{\text {int }}$ versus $\Delta E_{\text {int }}($ predicted $)=0.367 * E_{\mathrm{S}, \text { min }}-1.503 * V_{\mathrm{S}, \max }-0.352\left(E_{\mathrm{S}, \text { min }}\right.$ and $V_{\mathrm{S} \text {, max }}$ in $\mathrm{eV}$ ).

manner, provide complementary information for analyzing halogen bonding. To further explore this complementarity and how the relative importance of $V_{\mathrm{S}, \max }$ versus $E_{\mathrm{S} \text {,min }}$ for determining the interaction strength depends on the character of the halogen bond acceptor will be the objective of a future study.

\section{CONCLUSIONS}

The local electron attachment energy $[E(\mathbf{r})]$ has been introduced with the objective of defining a local property that is analogous to the average local ionization energy $[\bar{I}(\mathbf{r})]$ but that reflects susceptibility toward interaction with nucleophiles rather than electrophiles. The functional form $E(\mathbf{r})$ is motivated based on Janak's theorem and the piecewise linear energy dependence of electron addition to atomic and molecular systems. Within the GKS-DFT method, only the virtual orbitals with negative eigenvalues contribute to $E(\mathbf{r})$.

Similarly to $\bar{I}(\mathbf{r}), E(\mathbf{r})$ is complementary to the electrostatic potential $[V(\mathbf{r})]$ in that it is best suited to analyze donoracceptor interactions with a significant component of charge transfer and polarization. The positions of the lowest values of 
$E(\mathbf{r})$ on isodensity surfaces are generally associated with regions that are susceptible for nucleophilic attack, and the magnitude of the minima $\left(E_{\mathrm{S}, \min }\right)$ at those sites often correlates quantitatively with the relative reactivity.

We have shown that for fluorinated aromatics the lowest $E_{S, \min }$ of the aromatic carbons reflects the positional selectivity for nucleophilic aromatic substitution and that the relative rate constants of the corresponding reactions correlate with $E_{\mathrm{S}, \min }$. In aromatic olefins that have the double bond conjugated with resonance-withdrawing groups, the lowest $E_{\mathrm{S} \text {, min }}$ is found at the $\beta$-carbon, which is the site most susceptible to nucleophilic addition. The lowest $E_{S \text {,min }}$ of substituted benzylidenemalonitriles and $\alpha$-nitrostilbenes shows high correlation with rate constants for conjugate addition of piperidine and $\mathrm{HOCH}_{2} \mathrm{CH}_{2} \mathrm{~S}^{-}$, respectively.

Finally, we have analyzed the $E_{S}(\mathbf{r})$ of halomethanes and halobenzenes. In these systems, the lowest $E_{\mathrm{S} \text {,min }}$ is found at the tip of the iodine, bromine, or chlorine, which is the interaction site for halogen bonding. There is a very good linear correlation between interaction energy $\left(\Delta E_{\text {int }}\right)$ for halogen bonding of the halomethanes to formaldehyde and the $E_{S \text {,min }}$. In this series of molecules, the halogen $V_{\mathrm{S} \text {, max }}$ provides a very similar picture as the halogen $E_{\mathrm{S}, \min }$, and there is a good linear correlation between the two descriptors. Also, for the halobenzenes there is a very high correlation between $\Delta E_{\text {int }}$ and $E_{\mathrm{S}, \mathrm{min}}$. However, the $E_{\mathrm{S}, \min }$ correlates less with $V_{\mathrm{S} \text {, max }}$ and the complementarity of the two surface properties is manifested by an excellent dual-variable correlation with $\Delta E_{\text {int. }}$. These results warrant further investigations on the generality of the correlation between halogen bond strength and $E_{S, \min }$ and the relative importance of charge transfer and polarization for halogen bonding.

\section{ASSOCIATED CONTENT}

\section{(5) Supporting Information}

The Supporting Information is available free of charge on the ACS Publications website at DOI: 10.1021/acs.jpca.6b10142.

Computed and experimental data in table format for the size consistency analysis. Interactions of benzylidene malononitrale (BMN) and $\alpha$-nitrostilbene with Michael acceptors as well as for the halogen bonding of fluorinated methyl and arene halides. Summary of the statistical analysis of this study. (PDF)

\section{AUTHOR INFORMATION}

\section{Corresponding Author}

*E-mail: tore@kth.se.

\section{ORCID ${ }^{\circ}$}

Tore Brinck: 0000-0003-2673-075X

\section{Present Address}

${ }^{\dagger}$ P.C.: Novartis, Novartis Sverige AB, Box 1150, 18311 Täby, Sweden.

\section{Notes}

The authors declare no competing financial interest.

\section{ACKNOWLEDGMENTS}

T.B. thanks the Swedish Research Council (VR) for financial support. J.H.S. is grateful to the School of Chemical Science and Engineering at KTH for an excellence stipend. Sam Solten is acknowledged for computational assistance.

\section{REFERENCES}

(1) Weiner, P. K.; Langridge, R.; Blaney, J. M.; Schaefer, R.; Kollman, P. Electrostatic Potential Molecular-Surfaces. Proc. Natl. Acad. Sci. U. S. A. 1982, 79, 3754-3758.

(2) Sjoberg, P.; Murray, J.; Brinck, T.; Politzer, P. Average Local Ionization Energies on the Molecular-Surfaces of Aromatic Systems as Guides to Chemical-Reactivity. Can. J. Chem. 1990, 68, 1440-1443.

(3) Sjoberg, P.; Politzer, P. Use of the Electrostatic Potential at the Molecular-Surface to Interpret and Predict Nucleophilic Processes. J. Phys. Chem. 1990, 94, 3959-3961.

(4) Horenstein, B. A.; Schramm, V. L. Correlation of the Molecular Electrostatic Potential Surface of an Enzymatic Transition-State with Novel Transition-State Inhibitors. Biochemistry 1993, 32, 9917-9925.

(5) Brinck, T.; Murray, J.; Politzer, P. Molecular-Surface Electrostatic Potentials and Local Ionization Energies of Group-V-VII Hydrides and Their Anions - Relationships for Aqueous and Gas-Phase Acidities. Int. J. Quantum Chem. 1993, 48, 73-88.

(6) Hagelin, H.; Murray, J. S.; Politzer, P.; Brinck, T.; Berthelot, M. Family-Independent Relationships between Computed Molecular Surface Quantities and Solute Hydrogen Bond Acidity/Basicity and Solute-Induced Methanol OH Infrared Frequency Shifts. Can. J. Chem. 1995, 73, 483-488.

(7) Ehresmann, B.; Martin, B.; Horn, A. H. C.; Clark, T. Local Molecular Properties and Their Use in Predicting Reactivity. J. Mol. Model. 2003, 9, 342-347.

(8) Bulat, F. A.; Toro-labbe, A.; Brinck, T.; Murray, J. S.; Politzer, P. Quantitative Analysis of Molecular Surfaces: Areas, Volumes, Electrostatic Potentials and Average Local Ionization Energies. J. Mol. Model. 2010, 16, 1679-1691.

(9) Murray, J. S.; Politzer, P. Correlations between the Solvent Hydrogen-Bond-donating Parameter Alpha and the Calculated Molecular-Surface Electrostatic Potential. J. Org. Chem. 1991, 56, 6715-6717.

(10) Brinck, T.; Murray, J.; Politzer, P. Surface Electrostatic Potentials of Halogenated Methanes as Indicators of Directional Intermolecular Interactions. Int. J. Quantum Chem. 1992, 44, 57-64.

(11) Brinck, T. Modified Interaction Properties Function for the Analysis and Prediction of Lewis Basicities. J. Phys. Chem. A 1997, 101, 3408-3415.

(12) Brinck, T. In Theoretical Organic Chemistry; Párkányi, C., Ed.; Theoretical and Computational Chemistry; Elsevier: 1998; Vol. 5, pp 51-93.

(13) Riley, K. E.; Murray, J. S.; Politzer, P.; Concha, M. C.; Hobza, P. $\mathrm{Br} \cdots \mathrm{O}$ Complexes as Probes of Factors Affecting Halogen Bonding: Interactions of Bromobenzenes and Bromopyrimidines with Acetone. J. Chem. Theory Comput. 2009, 5, 155-163.

(14) Riley, K. E.; Murray, J. S.; Fanfrlik, J.; Rezac, J.; Sola, R. J.; Concha, M. C.; Ramos, F. M.; Politzer, P. Halogen Bond Tunability II: The Varying Roles of Electrostatic and Dispersion Contributions to Attraction in Halogen Bonds. J. Mol. Model. 2013, 19, 4651-4659.

(15) Aakeröy, C. B.; Wijethunga, T. K.; Desper, J.; Đaković, M. Electrostatic Potential Differences and Halogen-Bond Selectivity. Cryst. Growth Des. 2016, 16, 2662-2670.

(16) Koopmans, T. Über Die Zuordnung von Wellenfunktionen und Eigenwerten zu Den Einzelnen Elektronen Eines Atoms. Physica 1934, $1,104-113$.

(17) Bulat, F. A.; Levy, M.; Politzer, P. Average Local Ionization Energies in the Hartree- Fock and Kohn- Sham Theories. J. Phys. Chem. A 2009, 113, 1384-1389.

(18) Brinck, T.; Murray, J.; Politzer, P.; Carter, R. A Relationship Between Experimentally Determined $\mathrm{pK}_{\mathrm{A}} \mathrm{s}$ and Molecular-Surface Ionization Energies for Some Azines and Azoles. J. Org. Chem. 1991, 56, 2934-2936.

(19) Liljenberg, M.; Brinck, T.; Herschend, B.; Rein, T.; Rockwell, G.; Svensson, M. Validation of a Computational Model for Predicting the Site for Electrophilic Substitution in Aromatic Systems. J. Org. Chem. 2010, 75, 4696-4705.

(20) Brown, J. J.; Cockroft, S. L. Aromatic Reactivity Revealed: Beyond Resonance Theory and Frontier Orbitals. Chem. Sci. 2013, 4, 1772. 
(21) Brinck, T.; Murray, J.; Politzer, P. Relationships Between the Aqueous Acidities of Some Carbon, Oxygen, and Nitrogen Acids and the Calculated Surface Local Ionization Energies of Their Conjugate Bases. J. Org. Chem. 1991, 56, 5012-5015.

(22) Clark, T. The Local Electron Affinity for Non-Minimal Basis Sets. J. Mol. Model. 2010, 16, 1231-1238.

(23) Kramer, C.; Beck, B.; Clark, T. A Surface-Integral Model for Log $\mathrm{P}_{\mathrm{ow}}$. J. Chem. Inf. Model. 2010, 50, 404.

(24) Güssregen, S.; Matter, H.; Hessler, G.; Müller, M.; Schmidt, F.; Clark, T. 3D-QSAR based on Quantum-Chemical Molecular Fields: Toward an Improved Description of Halogen Interactions. J. Chem. Inf. Model. 2012, 52, 2441-2453.

(25) Bauer, T.; Jäger, C. M.; Jordan, M. J.; Clark, T. A Multi-Agent Quantum Monte Carlo Model for Charge Transport: Application to Organic Field-Effect Transistors. J. Chem. Phys. 2015, 143, 044114.

(26) Janak, J. F. Proof That $\partial E / \partial n_{i}=\varepsilon_{i}$ in Density-Functional Theory. Phys. Rev. B: Condens. Matter Mater. Phys. 1978, 18, 7165-7168.

(27) Cohen, A. J.; Mori-Sánchez, P.; Yang, W. Fractional Charge Perspective on the Band Gap in Density-Functional Theory. Phys. Rev. B: Condens. Matter Mater. Phys. 2008, 77, 115123.

(28) Peach, M. J.; Teale, A. M.; Helgaker, T.; Tozer, D. J. Fractional Electron Loss in Approximate DFT and Hartree-Fock Theory. J. Chem. Theory Comput. 2015, 11, 5262-5268.

(29) Mori-Sánchez, P.; Cohen, A. J.; Yang, W. Many-Electron SelfInteraction Error in Approximate Density Functionals. J. Chem. Phys. 2006, 125, 201102.

(30) Baerends, E. J.; Gritsenko, O. V.; van Meer, R. The Kohn-Sham Gap, the Fundamental Gap and the Optical Gap: The Physical Meaning of Occupied and Virtual Kohn-Sham Orbital Energies. Phys. Chem. Chem. Phys. 2013, 15, 16408-16425.

(31) Zhao, Y.; Truhlar, D. G. Design of Density Functionals That are Broadly Accurate for Thermochemistry, Thermochemical Kinetics, and Nonbonded Interactions. J. Phys. Chem. A 2005, 109, 5656-5667.

(32) Foster, M. E.; Wong, B. M. Nonempirically Tuned RangeSeparated DFT Accurately Predicts both Fundamental and Excitation Gaps in DNA and RNA Nucleobases. J. Chem. Theory Comput. 2012, 8, 2682-2687.

(33) Whittleton, S. R.; Sosa Vazquez, X. A.; Isborn, C. M.; Johnson, E. R. Density-Functional Errors in Ionization Potential with Increasing System Size. J. Chem. Phys. 2015, 142, 184106.

(34) Langenaeker, W.; Demel, K.; Geerlings, P. Quantum-Chemical Study of the Fukui Function as a Reactivity Index: Part 2. Electrophilic Substitution on Mono-Substituted Benzenes. J. Mol. Struct.: THEOCHEM 1991, 234, 329-342.

(35) Anderson, J. S.; Melin, J.; Ayers, P. W. Conceptual DensityFunctional Theory for General Chemical Reactions, Including Those That are Neither Charge- Nor Frontier-Orbital-Controlled. 2. Application to Molecules where Frontier Molecular Orbital Theory Fails. J. Chem. Theory Comput. 2007, 3, 375-389.

(36) Parr, R. G.; Von Szentpaly, L.; Liu, S. B. Electrophilicity Index. J. Am. Chem. Soc. 1999, 121, 1922-1924.

(37) Frisch, M. J.; Trucks, G. W.; Schlegel, H. B.; Scuseria, G. E.; Robb, M. A.; Cheeseman, J. R.; Scalmani, G.; Barone, V.; Mennucci, B.; Petersson, G. A. et al. Gaussian 09, revision D.01; Gaussian, Inc.: Wallingford, CT, 2009;

(38) Pettersen, E. F.; Goddard, T. D.; Huang, C. C.; Couch, G. S.; Greenblatt, D. M.; Meng, E. C.; Ferrin, T. E. UCSF Chimera, a Visualization System for Exploratory Research and Analysis. J. Comput. Chem. 2004, 25, 1605-1612.

(39) Leach, A. R. Molecular Modeling: Principles and Applications; Pearson Education, Ltd.: Harlow, England, 2001.

(40) Tanaka, K.; Deguchi, M.; Iwata, S. Ab initio Study of Nucleophilic Aromatic Substitution of Polyfluorobenzene. J. Chem. Res., Synop. 1999, $528-529$.

(41) Chambers, R. D.; Close, D.; Musgrave, W. K. R.; Waterhouse, J. S.; Williams, D. Mechanisms for Reactions of Halogenated Compounds.2. Orienting Effects of Chlorine Substituents in Nucleophilic Aromatic-Substitution. J. Chem. Soc., Perkin Trans. 2 1977, 1774-1778.
(42) Chambers, R. D.; Martin, P. A.; Waterhouse, J. S.; Williams, D. L. H.; Anderson, B. Mechanisms for Reactions of Halogenated Compounds.4. Activating Influences of Ring-Nitrogen and Trifluoromethyl in Nucleophilic Aromatic-Substitution. J. Fluorine Chem. 1982, 20, 507-514.

(43) Liljenberg, M.; Brinck, T.; Rein, T.; Svensson, M. Utilizing the Sigma-Complex Stability for Quantifying Reactivity in Nucleophilic Substitution of Aromatic Fluorides. Beilstein J. Org. Chem. 2013, 9, 791799.

(44) Brinck, T.; Liljenberg, M. In Arene Chemistry: Reaction Mechanisms and Methods of Aromatic Compounds; Mortier, J., Ed.; John Wiley \& Sons, Inc: Hoboken, NJ, 2015; pp 83-105.

(45) Liljenberg, M.; Brinck, T.; Herschend, B.; Rein, T.; Tomasi, S.; Svensson, M. Predicting Regioselectivity in Nucleophilic Aromatic Substitution. J. Org. Chem. 2012, 77, 3262-3269.

(46) Bernasconi, C. F.; Killion, R. B. Nucleophilic-Addition to Olefins.24. Is there a Transition-State Imbalance in Malononitrile Anion Forming Reactions - Kinetics of Piperidine and Morpholine Addition to Substituted Benzylidenemalononitriles in Various $\mathrm{Me}_{2} \mathrm{SO}$-Water Mixtures. J. Org. Chem. 1989, 54, 2878-2885.

(47) Domingo, L. R.; Perez, P.; Contreras, R. Reactivity of the CarbonCarbon Double Bond Towards Nucleophilic Additions. A DFT Analysis. Tetrahedron 2004, 60, 6585-6591.

(48) Bernasconi, C. F.; Killion, R. B. Nucleophilic Additions to Olefins.23. High Intrinsic Rate-Constant and Large Imbalances in the Thiolate Ion Addition to Substituted Alpha-Nitrostilbenes. J. Am. Chem. Soc. 1988, 110, 7506-7512.

(49) Riley, K. E.; Murray, J. S.; Fanfrlík, J.; Rezáč, J.; Solá, R. J.; Concha, M. C.; Ramos, F. M.; Politzer, P. Halogen Bond Tunability I: The Effects of Aromatic Fluorine Substitution on the Strengths of Halogen-Bonding Interactions Involving Chlorine, Bromine, and Iodine. J. Mol. Model. 2011, 17, 3309-3318.

(50) Riley, K. E.; Hobza, P. Investigations into the Nature of Halogen Bonding Including Symmetry Adapted Perturbation Theory Analyses. J. Chem. Theory Comput. 2008, 4, 232-242.

(51) Clark, T.; Hennemann, M.; Murray, J. S.; Politzer, P. Halogen Bonding: The Sigma-Hole. J. Mol. Model. 2007, 13, 291-296.

(52) Politzer, P.; Murray, J. S.; Clark, T. Halogen Bonding: An Electrostatically-Driven Highly Directional Noncovalent Interaction. Phys. Chem. Chem. Phys. 2010, 12, 7748-7757.

(53) Rosokha, S. V.; Stern, C. L.; Ritzert, J. T. Experimental and Computational Probes of the Nature of Halogen Bonding: Complexes of Bromine-Containing Molecules with Bromide Anions. Chem. - Eur. J. 2013, 19, 8774-8788.

(54) Mitoraj, M. P.; Michalak, A. Theoretical Description of Halogen Bonding - an Insight Based on the Natural Orbitals for Chemical Valence Combined with the Extended-Transition-state Method (ETSNOCV). J. Mol. Model. 2013, 19, 4681-4688.

(55) Wang, C.; Danovich, D.; Mo, Y.; Shaik, S. On the Nature of the Halogen Bond. J. Chem. Theory Comput. 2014, 10, 3726-3737.

(56) Politzer, P.; Murray, J. S.; Clark, T. Mathematical Modeling and Physical Reality in Noncovalent Interactions. J. Mol. Model. 2015, 21, 52.

(57) Reed, A. E.; Curtiss, L. A.; Weinhold, F. Intermolecular Interactions from a Natural Bond Orbital, Donor-Acceptor Viewpoint. Chem. Rev. 1988, 88, 899-926. 